\title{
Entropy Measure for Planning, Prediction and Online Estimation in Biotechnological Brocesses ${ }^{\dagger}$
}

\author{
Arnas Survyla, Benas Kemesis and Renaldas Urniežius \\ The Department of Automation, Kaunas University of Technology, Kaunas, Lithuania \\ + Presented at the Entropy 2021: The Scientific Tool of the 21st Century, 5-7 May 2021; Available online: \\ https://sciforum.net/conference/Entropy2021/.
}

Published: 5 May 2021

Recently, a generic bioprocess gray box modeling approach [1] used entropy measure to plan the feeding solution profile. Multiple industrial experiments showed that such modeling is useful in cultivations with limited substrate feeding. The feeding profile served as a scaled approximation of the cumulative biomass profile. The cumulative glucose volume served as uncertainty to find the gray box model parameters in the feedback control scenarios. The numeric convex approach passed an analysis of its sensitivity to different initial computational conditions. The validation showed that the numeric routines were independent of the selected initial conditions. Such simplicity makes it useful for practical industrial applications. Maximization of entropy presented online estimation of biomass concentration in fed-batch cultures of four types of recombinant E.coli strains and Saccharomyces cerevisiae cells [2]. Practical experience disclosed that entropy is a relevant measure for both limited substrate feeding and dosed substrate feeding biotechnological processes. Moreover, the approach showed neither numeric nor structural model dependence on the strain type. Research progress revealed that entropy measure by the use of fundamental knowledge could make the general model (Luedeking-Piret) more common for technological use when estimating target protein, compared to a sophisticated artificial neural network (ANN) [3]. In fact, it replaces the ANN approach without compromising estimation accuracy.

Funding: This project has received funding from European Regional Development Fund (project No 01.2.2-LMTK-718-03-0039) under grant agreement with the Research Council of Lithuania (LMTLT).

\section{References}

1. Urniezius, R.; Galvanauskas, V.; Survyla, A.; Simutis, R.; Levisauskas, D. From Physics to Bioengineering: Microbial Cultivation Process Design and Feeding Rate Control Based on Relative Entropy Using Nuisance Time. Entropy 2018, 20, 779.

2. Urniezius, R.; Survyla, A.; Paulauskas, D.; Bumelis, V.A.; Galvanauskas, V. Generic estimator of biomass concentration for Escherichia coli and Saccharomyces cerevisiae fed-batch cultures based on cumulative oxygen consumption rate. Microb. Cell Fact. 2019, 18, 190.

3. Urniezius, R.; Survyla, A. Identification of Functional Bioprocess Model for Recombinant E. Coli Cultivation Process. Entropy 2019, 21, 1221.

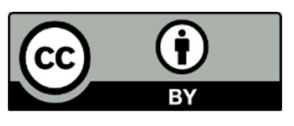

(C) 2021 by the authors. Licensee MDPI, Basel, Switzerland. This article is an open access article distributed under the terms and conditions of the Creative Commons Attribution (CC BY) license (http://creativecommons.org/licenses/by/4.0/). 